\title{
Pediatric neurosurgery during the COVID-19 pandemic: update and recommendations from the Brazilian Society of Pediatric Neurosurgery
}

\author{
*Matheus Fernando Manzolli Ballestero, MD, MSc, ${ }^{1,2}$ Luciano Furlanetti, MD, PhD, ${ }^{3}$ and \\ Ricardo Santos de Oliveira, MD, PhD²
}

${ }^{1}$ Department of Medicine, Federal University of São Carlos, Brazil; ²Division of Neurosurgery, Department of Surgery and Anatomy, University Hospital, Ribeirão Preto Medical School, University of São Paulo, Ribeirão Preto, Brazil; and ${ }^{3}$ Department of Neurosurgery, King's College Hospital Foundation Trust, Denmark Hill, London, United Kingdom

\begin{abstract}
OBJECTIVES Coronavirus disease (COVID-19) is a potentially severe respiratory illness that has threatened humanity globally. The pediatric neurosurgery practice differs from that of adults in that it treats children in various stages of physical and psychological development and contemplates diseases that do not exist in other areas. The aim of this study was to identify the level of knowledge and readiness of the healthcare providers, as well as to evaluate new preventive practices that have been introduced, psychological concerns, and the impact of the COVID-19 pandemic on pediatric neurosurgical units in Brazil.
\end{abstract}

METHODS Pediatric neurosurgeons were given an online questionnaire developed by the Brazilian Society of Pediatric Neurosurgery to evaluate the impact of the COVID-19 pandemic on their clinical practice.

RESULTS Of a cohort of 110 active members of the Brazilian Society of Pediatric Neurosurgery, 76 completed the survey (69\%). Ninety-six percent were aware of the correct use of and indication for the types of personal protective equipment in clinical and surgical practices, but only $73.7 \%$ of them had unrestricted access to this equipment. Ninetyeight percent of participants agreed or strongly agreed that the pandemic had affected their pediatric neurosurgical practice. The COVID-19 pandemic interfered with outpatient care in $88 \%$ of the centers, it affected neurosurgical activity in $90.7 \%$, and it led to the cancellation of elective neurosurgical procedures in $57.3 \%$. Concerning the impact of COVID-19 on surgical activity, $9.2 \%$ of the centers had less than $25 \%$ of the clinical practice affected, $46.1 \%$ had $26 \%-50 \%$ of their activity reduced, $35.5 \%$ had a $51 \%-75 \%$ reduction, and $9.2 \%$ had more than $75 \%$ of their surgical work cancelled or postponed. Sixty-three percent affirmed that patients had been tested for COVID-19 before surgery. Regarding the impact of the COVID-19 pandemic on the mental health of those interviewed, 3.9\% reported fear and anxiety with panic episodes, $7.9 \%$ had worsening of previous anxiety symptoms, $60.5 \%$ reported occasional fear, $10.5 \%$ had sadness and some depressive symptoms, and $2.6 \%$ reported depressive symptoms.

CONCLUSIONS The COVID-19 pandemic has posed unprecedented challenges to healthcare services worldwide, including neurosurgical units. Medical workers, pediatric neurosurgeons included, should be aware of safety measures and follow the recommendations of local healthcare organizations, preventing and controlling the disease. Attention should be given to the psychological burden of exposure to SARS-CoV-2 in healthcare workers, which carries a high risk of anxiety and depression.

https://thejns.org/doi/abs/10.3171/2020.9.FOCUS20703

KEYWORDS COVID-19; pediatric neurosurgery; pandemic

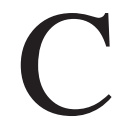
ORONAVIRUS disease (COVID-19) is a potentially severe respiratory illness that has threatened humanity globally. The first infected patients were identified in China in December 2019. COVID-19 is caused by a new coronavirus of the Coronaviridae family (SARSCoV-2), which is structurally related to other viruses re- sponsible for severe acute respiratory syndrome (SARS). ${ }^{1}$ Unlike the other 6 identified coronaviruses causing outbreaks during the last 18 years (e.g., SARS-CoV in 2002 and 2003, and MERS [Middle East respiratory syndrome] in 2012), ${ }^{2}$ the COVID-19 pandemic has become a major challenge for public health, research, and medical commu-

ABBREVIATIONS PPE = personal protective equipment; SARS = severe acute respiratory syndrome; SBNPed = Brazilian Society of Pediatric Neurosurgery SUBMITTED August 1, 2020. ACCEPTED September 23, 2020.

INCLUDE WHEN CITING DOI: 10.3171/2020.9.FOCUS20703.

* M.F.M.B., L.F., and R.S.d.O. contributed equally to this work. 
nities worldwide. SARS-CoV-2 has a high infection rate, a long incubation period, and a variety of manifestations mainly affecting the respiratory function, but also with neurological symptoms in some cases.

Evidence to date suggests that although children develop COVID-19, few will develop severe forms. ${ }^{3}$ However, fatal cases have already been described in some countries, such as the US, the UK, France, and Belgium, with an estimated mortality of $0.08 \%$ among the pediatric population. ${ }^{4}$

Although pediatric cases may be milder, children need greater hospital medical support and continuous assistance from their parents, generating a greater potential for transmissibility with nosocomial and community circulation of the virus. In addition, they can generate great social and financial burdens due to their parents' absence from their own activities. ${ }^{5}$

The role of pediatric neurosurgical care in global public health has only recently been widely recognized. ${ }^{6}$ A wide disparity in the access to pediatric neurosurgical care exists globally. In low-income and lower-middle-income countries, wherein there exists the greatest burden of pediatric neurosurgical disease, there is a known insufficiency of human and technological resources. ${ }^{6}$ The practice of pediatric neurosurgery differs from that of adults in that it treats children in various stages of physical and psychological development and contemplates diseases that do not exist in other areas.

In the face of the challenges posed by the COVID-19 pandemic, the Brazilian Society of Pediatric Neurosurgery (SBNPed) recommends that all elective, nonessential surgeries should be postponed whenever possible during the outbreak, mainly in the regions that are hit hardest and are classified as red flag areas.?

The aim of this study was to identify the level of knowledge and readiness of the healthcare providers, as well as to evaluate new preventive practices that have been introduced, psychological concerns, and the impact of the COVID-19 pandemic on pediatric neurosurgical units in Brazil. To the best of our knowledge, this is the first study to address the impact of the COVID-19 pandemic on pediatric neurosurgical practice.

\section{Methods}

Pediatric neurosurgeons were given an online questionnaire developed by the SBNPed, in order to evaluate the effects of the COVID-19 pandemic on their clinical practice. The study was conducted during the pandemic, from March 2020 to July 2020. The survey design and questions included were approved by the education committee and research advisory group of the SBNPed prior to distribution. Data regarding aspects such as main neurosurgical area of interest, years of clinical experience, psychological concerns regarding being exposed, availability of personal protective equipment (PPE), and the direct and indirect impact of the pandemic on surgical routine, among other variables, were collected (Table 1). All neurosurgeons willing to participate in the survey provided a written consent form authorizing the use and publication of the anonymized data for research. A convenient sampling method was used for data collection, and the distribution
TABLE 1. Survey on the impact of COVID-19 in pediatric neurosurgery in Brazil

\begin{tabular}{l}
\hline Main neurosurgical practice \\
\hline Spine neurosurgery \\
\hline Oncological neurosurgery \\
\hline Vascular neurosurgery \\
\hline Functional neurosurgery and pain \\
\hline Pediatric neurosurgery \\
\hline General neurosurgery \\
\hline How many years of experience in the area? \\
\hline Response: \\
\hline Are you working directly in the treatment of a patient with COVID-19 \\
(non-neurosurgical)? \\
\hline Yes \\
\hline No
\end{tabular}

How would you rate your feeling of fear/anxiety about the disease (you can choose more than one or none)?

Very fearful and anxious, with panic episodes

Previous anxiety worsens

Occasional fear

I'm not afraid

Sadness with some depressive symptoms

Depressive symptoms that hinder my professional life

I don't believe in pandemic

Do you know the indication for the use of each item of PPE in surgical, nursery, and outpatient situations?

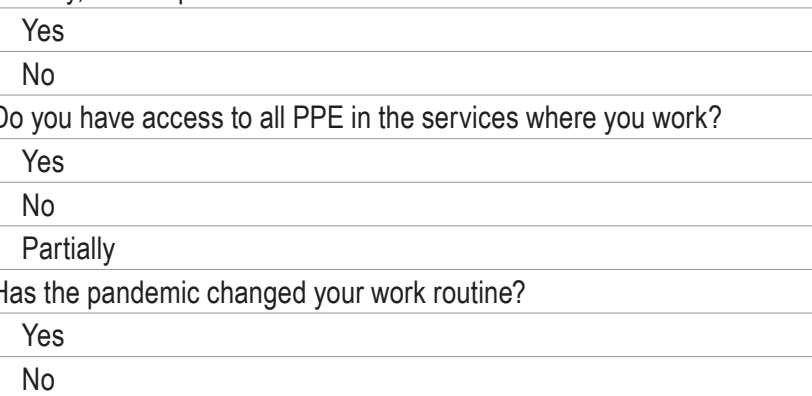

How has the pandemic changed your work routine (can choose more than one or none)?

Reduced outpatient capacity

Reduced number of surgical procedures

Dismissal from work for being in high-risk group

Cancellation of surgeries

Cancellation of other activities related to neurosurgery

What was the impact on your neurosurgical activity?

Less than $25 \%$

Between $26 \%$ and $50 \%$

Between $51 \%$ and $75 \%$

Over $75 \%$

In the main hospital where you work, was elective neurosurgery cancelled?

Yes

No 
》 CONTINUED FROM PAGE 2

TABLE 1. Survey on the impact of COVID-19 in pediatric neurosurgery in Brazil

Is there a test protocol for patients who will undergo neurosurgery? Yes

No

Have you ever tested positive for COVID-19 (sick or not)? Yes

No

Have you ever been sick with COVID-19 (not just a positive test)? Yes

No

Do you know of any official recommendations on surgical care during the pandemic?

Yes

No

Do you want to make additional comments about your involvement with the pandemic, your professional performance, or anything you consider important?

Response:

Authorization

I authorize the use of data from this research to compile tables and graphs that can be used for future scientific research. Under no circumstances will the identity of the respondents be disclosed. The participant can request that his/her answer be withdrawn from the survey at any time, even after the completion of the form. Select if you agree to participate in research.

I don't want to participate in this research.

of qualitative responses was presented as frequencies and percentages.

This study was approved by our institution's Research Advisory Group and informed consent was obtained from all members whose surveys were included.

\section{Results}

Of a cohort of 110 active members of the SBNPed, 76 completed the survey (69\% response rate). The participants' pediatric neurosurgical experience ranged from 1 to 45 years (mean 14.9 years, median 14 years, SD 11.2 years). Fifty-nine participants $(77.6 \%)$ reported pediatric neurosurgery as their main area of practice, and $81.6 \%$ were not directly involved with the management of hospitalized patients with COVID-19. Additionally, 73 participants $(96 \%)$ were aware of the correct use of and indication for the types of PPE in clinical and surgical practices. However, only $73.7 \%$ of them had the unrestricted access to PPE recommended by the WHO.

Ninety-eight percent (75 of 76) participants agreed or strongly agreed that the pandemic had affected their pediatric neurosurgical practice. Overall, the COVID-19 pandemic interfered with outpatient care in $88 \%$ of the centers, affected neurosurgical activity in $90.7 \%$, and led to the cancellation of elective neurosurgical procedures in $57.3 \%$. Four neurosurgeons (5.3\%) had been away from

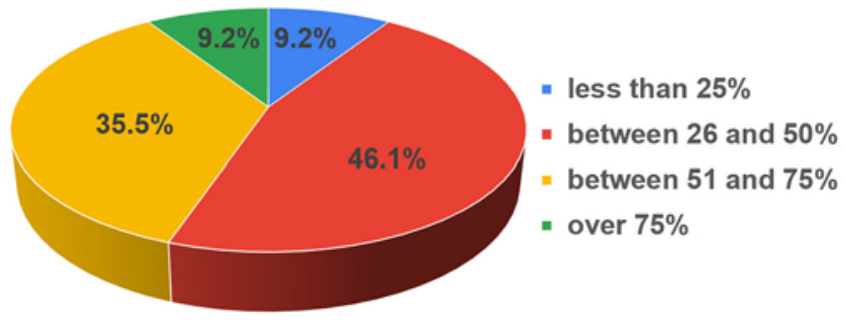

FIG. 1. Impact of COVID-19, in reductions of neurosurgical activity.

hospital activities since the beginning of the coronavirus outbreak because they belong to a high-risk group.

Concerning the impact of COVID-19 on surgical activity, $9.2 \%$ of the centers had less than $25 \%$ of the clinical practice affected, $46.1 \%$ had $26 \%-50 \%$ of their activity reduced, $35.5 \%$ had a $51 \%-75 \%$ reduction, and $9.2 \%$ had more than $75 \%$ of their surgical work cancelled or postponed (Fig. 1).

Sixty-three percent (48 of 76 respondents) affirmed that patients had been tested for COVID-19 before surgery. Interestingly, $81.6 \%$ of the interviewed neurosurgeons had tested negative for COVID-19 and $18.4 \%$ had tested positive, but only $11.9 \%$ presented clinical manifestation of the disease.

Figure 2 displays the current recommendations and workflow of the SBNPed for neurosurgeons during the COVID-19 pandemic. Regarding the impact of the COVID-19 pandemic on the mental health of interviewed pediatric neurosurgeons, $3.9 \%$ reported fear and anxiety with panic episodes, $7.9 \%$ had worsening of previous anxiety symptoms, $60.5 \%$ reported occasional fear, $10.5 \%$ had sadness and some depressive symptoms, and $2.6 \%$ reported depressive symptoms that were interfering with their professional life.

\section{Discussion}

The COVID-19 pandemic has become an international public health emergency, unprecedented in modern history. ${ }^{8}$ The SARS-CoV outbreak in 2003 had a much smaller impact than the current coronavirus pandemic. ${ }^{910}$ In terms of the number of deaths COVID-19 has caused (747,774 as of August 12, 2020; https://covid19.who.int), it is actually more comparable with previous flu pandemics. The H1N1 flu virus was first detected in April of 2009 in the US and spread quickly around the world. It was the first flu pandemic in 40 years, causing up to 575,400 dead in the first year the virus circulated. The pandemic was controlled in mid-October after a vaccine became available. ${ }^{11}$

In Brazil, the first COVID-19 patient was diagnosed in late February 2020. Unorganized governmental management of the coronavirus outbreak led the country to be ranked in second place worldwide in the number of infected people, surpassing 2.5 million confirmed cases. Brazil has officially registered more than 100,000 deaths to COVID-19, not to mention the high rate of undiagnosed and underreported cases (https://www.worldometers.info/ coronavirus/country/brazil). The transmission curve continues to rise almost exponentially amid disagreements 


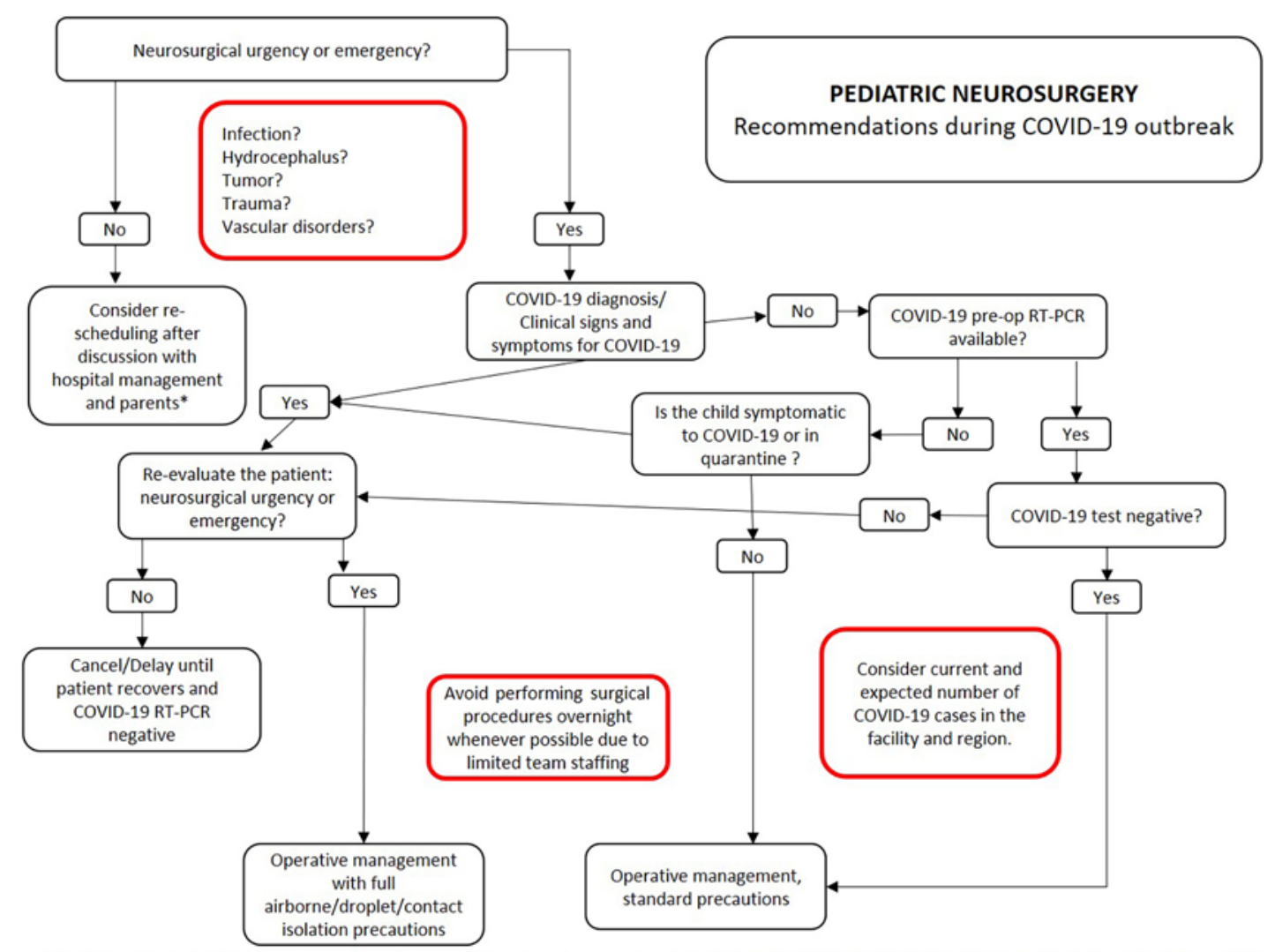

FIG. 2. SBNPed recommendations during COVID-19 outbreak. RT-PCR = reverse transcription polymerase chain reaction. *Elective surgeries and nonessential medical and surgical procedures should be delayed until the COVID-19 outbreak ends. Consider a range of factors when deciding whether to postpone a surgery or procedure, including patient risk factors, bed availability, staffing, and PPE supplies.

between authorities over the strategy to deal with the pandemic and its consequences. ${ }^{12}$

\section{Impact of the COVID-19 Pandemic on Healthcare Workers}

During the COVID-19 pandemic, as the world faces a shutdown or slowdown in daily activities and individuals are encouraged to implement social distancing, health professionals usually go in the opposite direction. Due to the exponential increase in the demand for health assistance, healthcare professionals face long work shifts, often with few resources and precarious infrastructure. The need for extra safety precautions and the use of uncomfortable PPE devices may also increase the physical and mental burden. ${ }^{13}$ In addition, many professionals may feel unprepared to carry out the clinical intervention in patients infected with a new virus, about which little is known, and for which there are no well-established clinical protocols or treatments so far. Also, there is the fear of autoinoculation, as well as the concern about the possibility of spreading the virus to their own families, friends, or colleagues.$^{14}$ This can lead healthcare workers to isolate themselves, change their routine, and narrow down their social support network. ${ }^{15,16}$

The psychological impact of social isolation and working with health services in a time of pandemic cannot be underestimated. García-Iglesias performed a systematic review to analyze the impact of the COVID-19 outbreak on healthcare professionals (nurses and doctors) and found medium to high levels of anxiety $(26.5 \%-44.6 \%)$ and depression $(8.1 \%-25 \%)$, as well as worry and insomnia, which were detected in $23.6 \%-38 \%$ of professionals. ${ }^{17}$ These results were compatible with the complaints of pediatric neurosurgeons included in the present study. In line with this, our survey evaluating the psychological impact of the COVID-19 pandemic on Brazilian pediatric neurosurgeons revealed a considerable prevalence of fear $(60.5 \%)$, anxiety (7.9\%), and also depressive symptoms $(10.5 \%)$, sometimes interfering with personal life (2.6\%).

\section{SBNPed Recommendations for Safe Neurosurgical Management of Pediatric Patients}

The incubation period of the SARS-CoV-2 ranges from 3 to 14 days; therefore, every patient presenting for treatment should be regarded as a potential asymptomatic infected case. ${ }^{18}$ The SBNPed, following the recommendations of the American College of Surgeons Clinical Issues and Guidance for the triage and management of elective surgical procedures (https://www.facs.org/covid-19/clinicalguidance), recommended that all elective, nonessential surgeries should be postponed during the outbreak of $\mathrm{CO}$ VID-19. ${ }^{7}$ Nasal endoscopic procedures are of special con- 


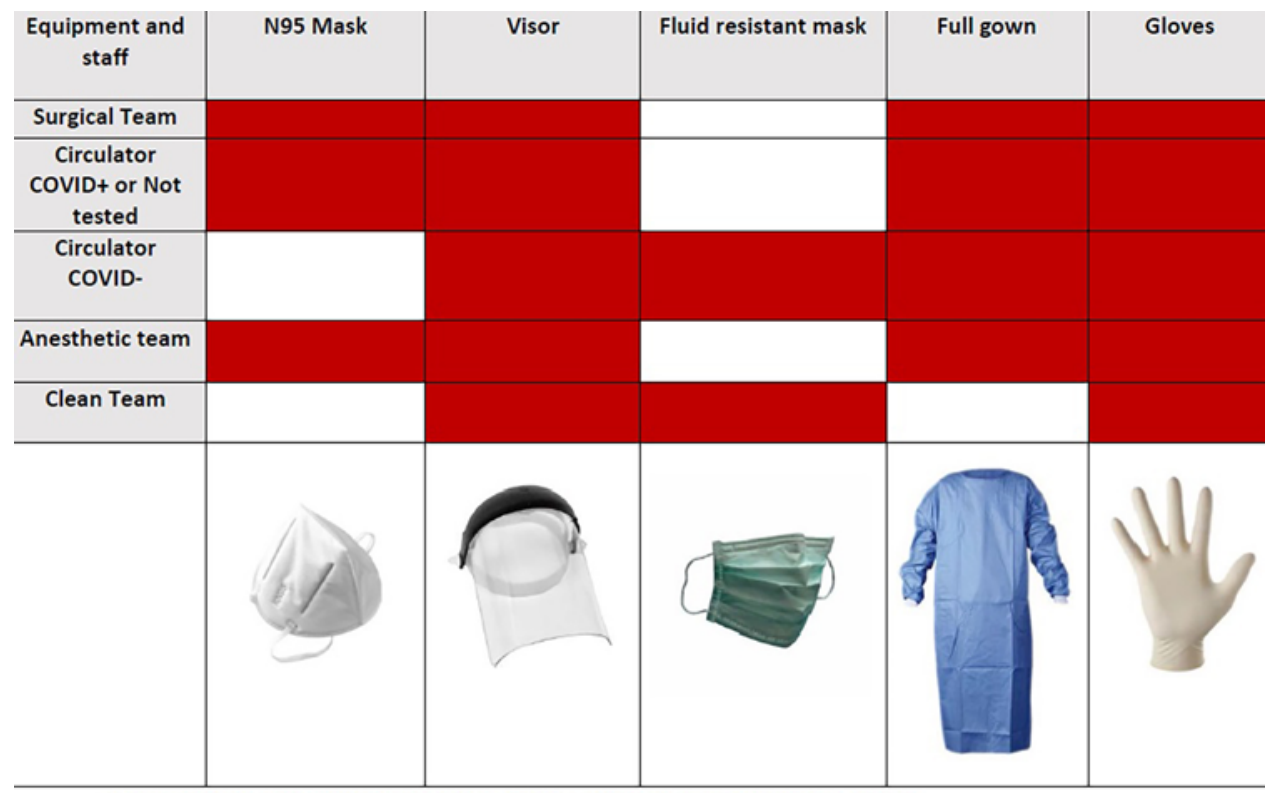

Always use a closed, waterproof and disposable surgical cap; Waterproof apron

FIG. 3. Summary on the use of PPE by the staff in the operating room.

cern and should be postponed whenever possible, mainly in regions of high risk affected by the pandemic. Nonessential hospital and office staff should be allowed to stay home and telework. ${ }^{19}$

Children presenting with raised intracranial pressure, intracranial and spinal tumors causing mass effect, acute hydrocephalus, infection, compression of vital structures, acute trauma, open dysraphism, or spinal and craniocervical instability must be considered for urgent or emergency surgical management. ${ }^{20}$ A summary of the SBNPed recommendations regarding procedures and time for surgery is presented in Appendix A.

Regarding PPE in the operation room, current guidelines vary depending on patient and institution COVID-19 status. ${ }^{21,22}$ Figure 3 summarizes the use of PPE by staff in the operating room recommended by the SBNPed.

\section{Conclusions}

The COVID-19 pandemic has posed unprecedented challenges to healthcare services worldwide, including neurosurgical units. Although the disease affects mainly adult patients, healthcare professionals dealing with the pediatric population are also exposed to the risk of acquiring and spreading the disease. Medical workers, pediatric neurosurgeons included, should be aware of safety measures and follow the recommendations of local healthcare organizations to prevent and control the disease. Attention should be given to the psychological burden of exposure to SARS-CoV-2 in healthcare workers, which carries a high risk of anxiety and depression. Further studies addressing this important but still underreported aspect of the COVID-19 pandemic are urgently needed.

\section{References}

1. Zhu N, Zhang D, Wang W, et al. A novel coronavirus from patients with pneumonia in China, 2019. $N$ Engl J Med. 2020;382(8):727-733.

2. de Wit E, van Doremalen N, Falzarano D, Munster VJ. SARS and MERS: recent insights into emerging coronaviruses. Nat Rev Microbiol. 2016;14(8):523-534.

3. Ludvigsson JF. Systematic review of COVID-19 in children shows milder cases and a better prognosis than adults. Acta Paediatr. 2020;109(6):1088-1095.

4. Liguoro I, Pilotto C, Bonanni M, et al. SARS-COV-2 infection in children and newborns: a systematic review. Eur $J$ Pediatr. 2020;179(7):1029-1046.

5. Bi Q, Wu Y, Mei S, et al. Epidemiology and transmission of COVID-19 in 391 cases and 1286 of their close contacts in Shenzhen, China: a retrospective cohort study. Lancet Infect Dis. 2020;20(8):911-919.

6. Dewan MC, Baticulon RE, Rattani A, et al. Pediatric neurosurgical workforce, access to care, equipment and training needs worldwide. Neurosurg Focus. 2018;45(4):E13.

7. de Oliveira RS, Ballestero MFM. The Covid-19 outbreak and pediatric neurosurgery guidelines. Arch Pediatr Neurosurg. 2020;2(1):53-54.

8. World Health Organization. Coronavirus disease (COVID-19) Weekly Epidemiological Update and Weekly Operational Update. Accessed October 12, 2020. https://www.who. int/emergencies/diseases/novel-coronavirus-2019/situationreports

9. Hung LS. The SARS epidemic in Hong Kong: what lessons have we learned? J R Soc Med. 2003;96(8):374-378.

10. Machhi J, Herskovitz J, Senan AM, et al. The natural history, pathobiology, and clinical manifestations of SARS-CoV-2 infections. J Neuroimmune Pharmacol. 2020;15(3):359-386.

11. Fineberg HV. Pandemic preparedness and response-lessons from the H1N1 influenza of 2009. N Engl J Med. 2014; 370(14):1335-1342.

12. Ribeiro F, Leist A. Who is going to pay the price of Covid-19? Reflections about an unequal Brazil. Int J Equity Health. 2020;19(1):91. 
13. Hall $\mathrm{H}$. The effect of the COVID-19 pandemic on healthcare workers' mental health. JAAPA. 2020;33(7):45-48.

14. Braquehais MD, Vargas-Cáceres S, Gómez-Durán E, et al. The impact of the COVID-19 pandemic on the mental health of healthcare professionals. QJM. 2020; hcaa207.

15. Ornell F, Schuch JB, Sordi AO, Kessler FHP. "Pandemic fear" and COVID-19: mental health burden and strategies. $\mathrm{Br}$ J Psychiatry. 2020;42(3):232-235.

16. Greenberg N. Mental health of health-care workers in the COVID-19 era. Nat Rev Nephrol. 2020;16(8):425-426.

17. García-Iglesias JJ, Gómez-Salgado J, Martín-Pereira J, et al. Impact of SARS-CoV-2 (Covid-19) on the mental health of healthcare professionals: a systematic review. Article in Spanish. Rev Esp Salud Publica. 2020;94:e202007088.

18. Lai CC, Shih TP, Ko WC, et al. Severe acute respiratory syndrome coronavirus 2 (SARS-CoV-2) and coronavirus disease-2019 (COVID-19): the epidemic and the challenges. Int J Antimicrob Agents. 2020;55(3):105924.

19. Prakash L, Dhar SA, Mushtaq M. COVID-19 in the operating room: a review of evolving safety protocols. Patient Saf Surg. 2020;14:30.

20. Weiner HL, Adelson PD, Brockmeyer DL, et al. Editorial. Pediatric neurosurgery along with Children's Hospitals' innovations are rapid and uniform in response to the COVID-19 pandemic. J Neurosurg Pediatr. 2020;26(1):3-5.

21. Ozoner B, Gungor A, Hasanov T, et al. Neurosurgical practice during coronavirus disease 2019 (COVID-19) pandemic. World Neurosurg. 2020;140:198-207.

22. American College of Surgeons. COVID-19: considerations for optimum surgeon protection before, during, and after operation. Accessed October 12, 2020. https://www.facs.org/ covid-19/clinical-guidance/surgeon-protection

\section{Disclosures}

The authors report no conflict of interest concerning the materials or methods used in this study or the findings specified in this paper.

\section{Author Contributions}

Conception and design: Ballestero, Santos de Oliveira. Acquisition of data: Ballestero, Santos de Oliveira. Analysis and interpretation of data: all authors. Drafting the article: all authors. Critically revising the article: all authors. Reviewed submitted version of manuscript: all authors. Approved the final version of the manuscript on behalf of all authors: Ballestero. Statistical analysis: Furlanetti. Administrative/technical/material support: Ballestero. Study supervision: Santos de Oliveira.

\section{Supplemental Information}

Online-Only Content

Supplemental material is available online. Appendix A. https://thejns.org/doi/suppl/10.3171/2020.9. FOCUS20703.

\section{Correspondence}

Matheus Fernando Manzolli Ballestero: University Hospital of Ribeirão Preto Medical School, University of São Paulo, Ribeirão Preto,Brazil.ballestero@gmail.com. 